\title{
Editorial (zbf Heft 2, 2015)
}

\section{Ferdinand Eder $\cdot$ Herbert Altrichter $\cdot$ Angelika Paseka}

Online publiziert: 23. Juli 2015

(C) Springer Fachmedien Wiesbaden 2015

Das vorliegende Heft der ,Zeitschrift für Bildungsforschung“versammelt sechs Beiträge, die sich mit sehr heterogenen Aspekten des Bildungswesens befassen: mit der Rekrutierung der künftigen Lehrpersonen, mit den demokratischen Orientierungen von Studierenden, mit dem Umgang der Schülerinnen und Schüler mit Lernstrategien und mit dem Fach Mathematik, aber auch mit der Gesundheitsförderung als gesellschaftliche Aufgabe der Schule ebenso wie mit der Frage, wie GovernanceProzesse im Bereich der Bildung für nachhaltige Entwicklung ablaufen.

Lehramtsstudierende, so der häufige Eindruck, stammen sehr oft aus Lehrerfamilien. Martin Rothland (Universität Siegen), Johannes König (Universität Köln) und Martin Drahmann (Universität Siegen) gehen in ihrem Beitrag „Lehrerkinder. Zur Bedeutung der Berufsvererbung für die Berufswahl Lehramt“ der Frage nach, ob dieser Eindruck gerechtfertigt ist. In einem Ländervergleich zwischen Deutschland, Österreich und der Schweiz zeigt sich, dass die Berufsvererbungsquote zwischen 20 und $30 \%$ liegt. Eine Analyse der Berufswahlmotive fördert interessante Ergebnisse im Hinblick auf die Einschätzung des zukünftigen Berufes zutage.

Sebastian Dippelhofer (Justus-Liebig-Universität Gießen) nutzt Querschnittdaten des deutschen bundesweiten Studierendensurveys, um „Politisch-demokratische Orientierungen und hochschulpolitisches Engagement von Studierenden“ zu analysieren. Dabei ergibt sich ein Bild einer in universitätsbezogenen Fragen wenig aktiv

F. Eder $(\bowtie)$

Paris Lodron Universität Salzburg,

Salzburg, Österreich

E-Mail: ferdinand.eder@sbg.ac.at

H. Altrichter

Johannes Kepler Universität Linz,

Linz, Österreich

A. Paseka

Universität Hamburg,

Hamburg, Deutschland 
engagierten und gegenüber politisch-demokratischen Werten zunehmend ambivalenten und indifferenten Studierendenschaft. Auf der anderen Seite zeigt sich aber ein Interesse an allgemein-politischen Themen und die Bereitschaft, trotz des geringen Engagements konkrete - auch politische - Ziele zu formulieren. In multivariaten Analysen erkennt der Autor ein durchaus bestehendes Potential der Universitäten, Studierende demokratisch zu stärken.

Der Beitrag von Yves Karlen (Universität Zürich) über „Nutzungshäufigkeit von Lernstrategien und metakognitives Strategiewissen in der Oberstufe des Gymnasiums“" untersucht an einer großen Stichprobe von Zürcher Gymnasiastinnen und Gymnasiasten die Entwicklung von Lernstrategien und des metakognitiven Strategiewissens innerhalb eines Schuljahres. Dabei zeigen sich Veränderungen für die Nutzungshäufigkeit von Elaborations-, Planungs- und Evaluationsstrategien, nicht jedoch für Transformationsstrategien und für metakognitives Strategiewissen. Auch wird deutlich, dass die Nutzungshäufigkeit von Lernstrategien keinen Einfluss auf die Entwicklung des metakognitiven Strategiewissens hat, das letztere jedoch Transformations- und Evaluationsstrategien beeinflusst.

Mathematik gilt als eines der unbeliebtesten Schulfächer. Tina Hascher (Universität Bern) und Sabine Reindl (Pädagogische Hochschule Linz) versuchen in ihrem Beitrag „Einstellung von Grundschulkindern zum Schulfach Mathematik“ zu klären, ob das schon zu Beginn der Schullaufbahn so ist und wie sich diese Einstellungen entwickeln. Ihre Ergebnisse weisen darauf hin, dass Schulanfänger/innen einen durchaus positiven Bezug zur Mathematik haben, der sich jedoch im Laufe der Grundschulzeit verringert. Ein Ergebnis, das zu denken gibt.

In ihrem Beitrag „Gesundheit im Kontext von Bildung und Erziehung: Entwicklung, Umsetzung und Herausforderungen der schulischen Gesundheitsförderung in Deutschland“" geben Kevin Dadaczynski, Peter Paulus, Birgit Nieskens und Heinz Hundeloh (Leuphana Universität Lüneburg) einen umfassenden Überblick über den Stand und die Entwicklung der schulischen Gesundheitsförderung in Deutschland. Aus der Analyse von Praxiserfahrungen und des aktuellen Fachdiskurs werden einige Herausforderungen abgeleitet, die sich der schulischen Gesundheitsförderung zukünftig stellen.

Jutta Nikel und Christoph Haker (Pädagogische Hochschule Freiburg) untersuchen in ihrem Beitrag „Intermediäre Aushandlungsräume“ die Steuerung und Koordination von Entwicklungsprozessen in den deutschen Bundesländern im Zuge der Weltdekade „Bildung für nachhaltige Entwicklung“ der Vereinten Nationen. Das Beispiel ist für die Diskussion von Governance-Prozessen insofern gut geeignet, weil unterschiedlich verfasste Akteure zwischen Zivilgesellschaft und Staat beteiligt sind. Die Qualität der intermediären Aushandlungsräume im Zuge dieser Prozesse wird mit sechs analytische Dimensionen beschrieben.

Josef Thonhauser betreut seit dem ersten Erscheinen der ZBF im Jahre 2011 den Bereich „Rezensionen“ und liefert dazu mit bewundernswerter Regelmäßigkeit Besprechungen pädagogischer Arbeiten, die nicht nur wegen ihres Inhalts, sondern auch wegen ihres pointierten Stils kleine Sternstunden im Lesealltag darstellen. $\mathrm{Zu}$ unserem großen Bedauern hat er mitgeteilt, dass er diese Aufgabe nunmehr aus Altersgründen beendet. Es ist uns als Herausgeber/innen ein großes Bedürfnis, ihm für seine Tätigkeit sehr herzlich zu danken. 
In diesem Heft erscheint damit seine letzte Rezension und sie behandelt ein ungewöhnliches Buch: Alma de Zarate, Jamila Tressel \& Lara-Luna Ehrenschneider in Zusammenarbeit mit Uli Hauser (2014). Wie wir Schule machen. Lernen, wie es uns gefällt. München: Knaus, 191 Seiten.

Und wie immer wird das Heft durch Nachrichten aus der ÖFEB - diesmal über eine Veranstaltung der Emerging Researcher - vervollständigt. 